\title{
Bedaquiline for the treatment of multidrug-resistant tuberculosis: another missed opportunity?
}

\author{
Lorenzo Guglielmetti (10) ${ }^{1,2,3}$
}

\begin{abstract}
Affiliations: ${ }^{1}$ Médecins Sans Frontières, Paris, France. ${ }^{2}$ APHP, Centre National de Référence des Mycobactéries et de la Résistance des Mycobactéries aux Antituberculeux, Bactériologie-Hygiène, Hôpitaux Universitaires Pitié Salpêtrière-Charles Foix, Paris, France. ${ }^{3}$ Sorbonne Université, UPMC Université Paris 06 , CR7, INSERM, U1135, Centre d'Immunologie et des Maladies Infectieuses, CIMI, Team E13 (Bactériologie),
\end{abstract} Paris, France.

Correspondence: Lorenzo Guglielmetti, Sorbonne Université, UPMC Université Paris 06, CR7, INSERM, U1135, Centre d'Immunologie et des Maladies Infectieuses, CIMI, Team E13 (Bactériologie), Laboratoire de Bactériologie-Hygiène, Faculté de Médecine Pierre et Marie Curie (UPMC Paris 6), 91 Boulevard de l'hôpital, Paris 75634, France. E-mail: lorenzo.guglielmetti@gmail.com

@ERSpublications

Scale-up of introduction of bedaquiline, as part of optimised treatment regimens, is supported by growing evidence http://ow.ly/JCEV30aPLmq

Cite this article as: Guglielmetti L. Bedaquiline for the treatment of multidrug-resistant tuberculosis: another missed opportunity? Eur Respir J 2017; 49: 1700738 [https://doi.org/10.1183/13993003.007382017].

5 years after its first approval by a stringent regulatory authority, the role of bedaquiline in the treatment of multidrug-resistant tuberculosis (MDR-TB) and extensively drug-resistant tuberculosis (XDR-TB) is well established, and acknowledged by existing guidance. However, in many regards, the global introduction of bedaquiline can hardly be considered as a success.

Bedaquiline, an innovative compound belonging to the diarylquinoline family, was developed in 2005 [1], and approved by the Food and Drug Administration and the European Medicines Agency in 2012 and 2013, respectively. The results of two phase IIb clinical trials have shown promising results when adding bedaquiline to an optimised background MDR-TB regimen [2-5], and raised new hopes in a field, like drug-resistant TB treatment, which had not seen any relevant innovation for decades. However, except for a few countries leading the way in the early programmatic introduction of bedaquiline [6], the global roll-out of this drug has been inadequate. According to a report by the Drug-Resistant TB Scale-up Treatment Action Team (DR-TB STAT), no more than 8195 patients globally were reported to have received treatment with bedaquiline before March 2017, with the large majority of them (59.6\%) being treated in only one country, South Africa [7]. The disproportion between these numbers and the need for bedaquiline is striking: according to estimates provided by a multicentre study that included MDR-TB patients from the World Health Organization's (WHO) Europe and Africa regions, approximately one-third of patients would be eligible for treatment with bedaquiline based on drug-resistance criteria [8]. Moreover, the proportion of eligible patients exceeds two-thirds of all cases if we include those with baseline risk factors for poor outcome, as suggested by the recently updated WHO recommendations on the use of bedaquiline [9].

Unfortunately, the slow pace of the introduction of bedaquiline has not prevented the appearance of strains with resistance to this drug [10-12]. Although drug resistance has inevitably followed the

Received: April 102017 | Accepted after revision: April 102017

Conflict of interest: Disclosures can be found alongside this article at erj.ersjournals.com

Copyright @ERS 2017 
introduction of new antibiotics in the history of antimicrobial chemotherapy, some of the practices that are currently established in the use of bedaquiline may arguably increase the risk of resistance selection [13]. In most settings, bedaquiline is used for 24 weeks as a component of conventional regimens, which usually last 20-24 months. The standardised duration of bedaquiline treatment is recommended by the manufacturer according to the evidence provided by clinical trials where bedaquiline was given for 24 weeks. This recommendation does not take into account the specific needs of individual MDR-TB patients. The interruption of bedaquiline treatment may lead to the administration of unacceptably weak regimens in cases where no therapeutic alternatives are available. A recent study confirms these concerns: in the study, almost $20 \%$ of 53 MDR-TB patients who had achieved sputum culture conversion with bedaquiline-containing regimens experienced reversion to positive cultures after interrupting bedaquiline treatment at 24 weeks [14]. In addition, current recommendations warn against the concomitant use of the new drugs, bedaquiline and delamanid, on the grounds of a lack of evidence and the potential for a synergic increase in QT interval prolongation. Even if based on understandable safety concerns, an excess of prudence in this case may lead to prioritising the prevention of the virtual risk of cardiac toxicity over the well-documented risk of death associated with drug-resistant TB. Indeed, the association of bedaquiline and delamanid may represent the only chance of an effective treatment for XDR-TB cases with advanced resistance patterns or intolerance to multiple drugs. The sequential use of the new drugs, after a recommended wash-out period of 6 months when switching from bedaquiline to delamanid, contradicts the principles of anti-TB treatment and may increase the risk of acquisition of drug resistance, as exemplified by a case report in which resistance to bedaquiline and delamanid was acquired sequentially during treatment $[12,15,16]$.

The shortcomings in global access to bedaquiline are reflected by the paucity of available evidence $[17,18]$. Only a few treatment cohorts including at least 10 patients have been published to date [19-23], and only one reporting treatment outcomes [24]. Extended treatment duration with bedaquiline has so far been described in one small cohort and in a case report $[24,25]$, and only two case reports of concurrent treatment with bedaquiline and delamanid have been published [26, 27].

These research gaps emphasise the importance of the study by Borisov et al. [28] presented in this issue of the European Respiratory Journal, which reports the results of the first large multicentre cohort of patients receiving bedaquiline-containing treatment. This retrospective cohort study involved 25 centres from 15 different countries, including settings with both high and low TB burden. Consecutive, culture-confirmed MDR-TB patients treated outside clinical trials with a regimen including bedaquiline in the period between 2008 and 2016 were included in the study, regardless of the timing of bedaquiline introduction. Treatment efficacy was assessed through sputum culture conversion rates and, for patients who ended treatment, the outcomes of the treatment. Treatment safety data was collected, including QT interval follow-up when available, although no standardised protocol to grade severity and seriousness of the adverse events, nor their causality link with bedaquiline, was implemented. Overall, 428 MDR-TB patients were included, mostly from South Africa (44\%) and the Russian Federation (28\%). The cohort includes high rates of XDR-TB (46\%) and previously treated (78\%) cases, and a total prevalence of HIV infection of $22 \%$ among those tested. Treatment regimens included, in more than half of cases, re-purposed drugs like linezolid and clofazimine. Sputum culture conversion rates were $57 \%$ and $81 \%$ at 2 and 3 months after anti-TB treatment commenced, respectively. Remarkably, $77 \%$ of the 130 patients in the 2008-2014 cohort, which included patients with $\geqslant 2$ years of follow-up, achieved favourable treatment outcomes. These figures appear to be consistent with the outcomes of patients starting treatment after 2014, although the results of these recent cohorts are preliminary and should be interpreted carefully. As expected with MDR-TB regimens, a high rate of adverse events occurred during treatment. Treatment with bedaquiline was interrupted in $12 \%$ of patients, and discontinued permanently in $6 \%$ : the reasons for discontinuation were not reported. QT interval corrected according to the Fridericia formula (QTcF) values of $>500 \mathrm{~ms}$ were recorded in 24 out of 247 (9.7\%) patients with available data. Among the patients who died and had available QT follow-up information, no one experienced QT prolongation, with the exception of one patient who already had $>500 \mathrm{~ms}$ QTcF at baseline. One patient died after developing a severe ventricular arrhythmia, although his QTcF never exceeded $500 \mathrm{~ms}$.

Overall, this collaborative study provides us with a wealth of evidence on the use of bedaquiline. First of all, the efficacy results are remarkable. The rate of favourable outcomes in the cohort who commenced treatment before 2014 is in line with the upper boundary of the confidence interval of the meta-analysis included in the latest WHO recommendations [9], and is much higher than those reported in systematic reviews of MDR/XDR-TB patients treated without new and repurposed drugs [8, 29, 30]. Early culture conversion rates are also high, although the inclusion of patients starting bedaquiline at different time-points during treatment makes it difficult to single out the role of bedaquiline. Although prone to the shortcomings of retrospective, non-standardised data collection, safety results are reassuring. This is in line 
with other published reports $[19,22-24]$ and with the conclusions of the recently revised WHO recommendations, which downgraded the anticipated undesirable effects of bedaquiline from "large" to "moderate" [9].

In this framework, the study by BoRIsov et al. [28] represents a source of information that should not be overlooked. The authors' encouraging results bring additional evidence to support a systematic introduction of bedaquiline for patients who need it. At the same time, they highlight the urgent need for well-documented prospective observational cohorts of patients receiving bedaquiline in the years before results from phase III clinical trials will become available. The timely roll-out of bedaquiline will require, in parallel, increased attention to prevent drug resistance, including optimal, individualised patient management. The use of prolonged bedaquiline treatment and the combination of bedaquiline and delamanid are therapeutic options that should be considered whenever indicated. Criteria have already been proposed to guide the selection of such patients $[24,31]$. This is the time to scale-up and improve our efforts to avoid bedaquiline being remembered as another missed opportunity in the history of $\mathrm{TB}$ control.

\section{References}

1 Andries K, Verhasselt P, Guillemont J, et al. A diarylquinoline drug active on the ATP synthase of Mycobacterium tuberculosis. Science 2005; 307: 223-227.

2 Diacon AH, Pym A, Grobusch MP, et al. Multidrug-resistant tuberculosis and culture conversion with bedaquiline. N Engl J Med 2014; 371: 723-732.

3 Pym AS, Diacon AH, Tang SJ, et al. Bedaquiline in the treatment of multidrug- and extensively drug-resistant tuberculosis. Eur Respir J 2016; 47: 564-574.

4 Diacon AH, Pym A, Grobusch M, et al. The diarylquinoline TMC207 for multidrug-resistant tuberculosis. $N$ Engl J Med 2009; 360: 2397-2405.

5 Diacon AH, Donald PR, Pym A, et al. Randomized pilot trial of eight weeks of bedaquiline (TMC207) treatment for multidrug-resistant tuberculosis: long-term outcome, tolerability, and effect on emergence of drug resistance. Antimicrob Agents Chemother 2012; 56: 3271-3276.

6 Guglielmetti L, Hewison C, Avaliani Z, et al. Examples of bedaquiline introduction for the management of multidrug-resistant tuberculosis in five countries. Int J Tuberc Lung Dis 2017; 21: 167-174.

$7 \quad$ DR-TB STAT. http://drtb-stat.org/global-snapshot/ Date last updated: March 2017.

8 Bonnet $\mathrm{M}$, Bastard $\mathrm{M}$, du Cros $\mathrm{P}$, et al. Identification of patients who could benefit from bedaquiline or delamanid: a multisite MDR-TB cohort study. Int J Tuberc Lung Dis 2016; 20: 177-186.

9 World Health Organization. Report of the Guideline Development Group Meeting on the use of bedaquiline in the treatment of multidrug-resistant tuberculosis: a review of available evidence (2016). WHO/HTM/TB/2017.01. Geneva, WHO, 2017.

10 Veziris N, Bernard C, Guglielmetti L, et al. Rapid emergence of Mycobacterium tuberculosis bedaquiline resistance: lessons to avoid repeating past errors. Eur Respir J 2017; 49: 1601719.

11 Andries K, Villellas C, Coeck N, et al. Acquired resistance of Mycobacterium tuberculosis to bedaquiline. PLoS ONE 2014; 9: e102135.

12 Bloemberg GV, Keller PM, Stucki D, et al. Acquired resistance to bedaquiline and delamanid in therapy for tuberculosis. N Engl J Med 2015; 373: 1986-1988.

13 Guglielmetti L, Le Dû D, Fréchet-Jachym M, et al. Preventing acquired resistance to bedaquiline and delamanid in multidrug-resistant tuberculosis treatment requires optimal management. Am J Respir Crit Care Med 2016; 194: $1170-1171$

14 Kiria N, Khachatryan N. Compassionate use of bedaquiline in Armenia and Georgia: end of treatment results. http://www.msf-tb-symposium.org/files/4414/8941/4754/5.2_Nana_Kiria_Naira_Khachatryan_Compassionate_ use_of_Bdq_ENG.pdf Date last accessed: May 3, 2017. Date last updated: March 2017.

15 Somoskovi A, Bruderer V, Hömke R, et al. A mutation associated with clofazimine and bedaquiline cross-resistance in MDR-TB following bedaquiline treatment. Eur Respir J 2015; 45: 554-557.

16 Hoffmann H, Kohl TA, Hofmann-Thiel S, et al. Delamanid and bedaquiline resistance in Mycobacterium tuberculosis ancestral Beijing genotype causing extensively drug-resistant tuberculosis in a Tibetan refugee. Am J Respir Crit Care Med 2016; 193: 337-340.

17 Pontali E, Sotgiu G, D'Ambrosio L, et al. Bedaquiline and MDR-TB: a systematic and critical analysis of the evidence. Eur Respir J 2016; 47: 394-402.

18 Pontali E, D'Ambrosio L, Centis R, et al. Multidrug-resistance tuberculosis and beyond: an updated analysis of the current evidence on bedaquiline. Eur Respir J 2017; 49: 1700146.

19 Skrahina A, Hurevich H, Falzon D, et al. Bedaquiline in the multidrug-resistant tuberculosis treatment: Belarus experience. Int J Mycobacteriol 2016; 5: Suppl. 1, S62-S63.

20 Guglielmetti L, Le Dû D, Jachym M, et al. Compassionate use of bedaquiline for the treatment of multidrug-resistant and extensively drug-resistant tuberculosis: interim analysis of a French dohort. Clin Infect Dis 2015; 60: 188-194.

21 Guglielmetti L, Le Dû D, Veziris N, et al. Is bedaquiline as effective as fluoroquinolones in the treatment of multidrug-resistant tuberculosis? Eur Respir J 2016; 48: 582-585.

22 Ndjeka N, Conradie F, Schnippel K, et al. Treatment of drug-resistant tuberculosis with bedaquiline in a high HIV prevalence setting: an interim cohort analysis. Int J Tuberc Lung Dis 2015; 19: 979-985.

23 Udwadia ZF, Ganatra S, Mullerpattan JB. Compassionate use of bedaquiline in highly drug-resistant tuberculosis patients in Mumbai, India. Eur Respir J 2017; 49: 1601699.

24 Guglielmetti L, Jaspard M, Le Dû D, et al. Long-term outcome and safety of prolonged bedaquiline treatment for multidrug-resistant tuberculosis. Eur Respir J 2017; 49: 1601799. 
25 Lewis JM, Hine P, Walker J, et al. First experience of effectiveness and safety of bedaquiline for 18 months within an optimised regimen for XDR-TB. Eur Respir J 2016; 47: 1581-1584.

26 Lachâtre M, Rioux C, Le Dû D, et al. Bedaquiline plus delamanid for XDR tuberculosis. Lancet Infect Dis 2016; 16: 294.

27 Tadolini M, Lingtsang RD, Tiberi S, et al. First case of extensively drug-resistant tuberculosis treated with both delamanid and bedaquiline. Eur Respir J 2016; 48: 935-938.

28 Borisov SE, Dheda K, Enwerem M, et al. Effectiveness and safety of bedaquiline-containing regimens in the treatment of MDR- and XDR-TB: a multicentre study. Eur Respir J 2017; 49: 1700387.

29 Falzon D, Gandhi N, Migliori GB, et al. Resistance to fluoroquinolones and second-line injectable drugs: impact on multidrug-resistant TB outcomes. Eur Respir J 2013; 42: 156-168.

30 Jacobson KR, Tierney DB, Jeon CY, et al. Treatment outcomes among patients with extensively drug-resistant tuberculosis: systematic review and meta-analysis. Clin Infect Dis 2010; 51: 6-14.

31 Matteelli A, D'Ambrosio L, Centis R, et al. Compassionate and optimum use of new tuberculosis drugs. Lancet Infect Dis 2015; 15: 1131-1132. 